\title{
Polydimethylsiloxane Resin Nanocomposite Coating with Alternating Multilayer Structure for Corrosion Protection Performance
}

\author{
Jianping Liu, ${ }^{1}$ Jiaoxia Zhang, ${ }^{1,}$ Jijun Tang,,${ }^{1 *}$ Liuyue Pu, ${ }^{1}$ Yanli Xue, ${ }^{2}$ Meiqun Lu, ${ }^{1}$ Lei Xu ${ }^{4}$ and Zhanhu Guo ${ }^{3, *}$
}

\begin{abstract}
Polydimethylsiloxane (PDMS) composite coatings filled with nanoparticles have been used extensively as anticorrosion materials. The alternating multilayer polydimethylsiloxane resin nanocomposite coating was first fabricated based on stepwise coating method via adding graphene and $\alpha$-alumina. Their mechanical and anticorrosion properties were discussed. The binary fillers greatly improved the adhesion up to level 1, at the same time, the coating remained extreme flexibility. When the graphene was $0.3 \mathrm{wt} \%$, the alternating multilayer coating exhibited the best corrosion resistance with the $4.82 \mathrm{~V}$ of corrosion potential ( $\mathrm{E}_{\text {corr }}$ ), which was increased by near 40 times compared with pure PDMS coating. Meanwhile, the impedance value of composite coating reached $10^{9} \Omega \bullet \mathrm{cm}^{2}$. The corrosion current density ( $\mathrm{I}_{\text {corr }}$ ) of the coating was smaller than that of bare tinplate. The long-term soaking results prove that the alternating multilayer composite coating still possess the outstanding corrosion resistance for tinplate after 30-day immersion.
\end{abstract}

Keywords: Alternating layered structure; Polydimethylsiloxane; Graphene; $\alpha$-alumina; Composite coating; Anticorrosion.

Received: 15 May 2020; Accepted: 03 September 2020.

Article type: Research article.

\section{Introduction}

Corrosion refers to the process of loss and destruction caused by the chemical or electrochemical interaction between metals substances and surrounding environmental mediums, which lead to the deterioration and disintegration of metals or their alloy materials..$^{[1-3]}$ Corrosion exists in all industries and can be seen from ships, bridges, houses and even well-

\footnotetext{
${ }^{1}$ School of Materials Science and Engineering, Jiangsu University of Science and Technology, Zhenjiang 212003, China.

${ }^{2}$ Road Environment Science and technology CO., LTD, Wuhan 430000, China.

3 Integrated Composites Laboratory (ICL), Department of Chemical \& Biomolecular Engineering, University of Tennessee, Knoxville, TN 37996 USA.

${ }^{4}$ School of Materials Science and Engineering, Shaanxi University of Technology, Hanzhong 723001, China.

*Email: zhangjx@just.edu.cn (J. Zhang); zguo10@utk.edu (Z. Guo).
}

preserved precision instruments. ${ }^{[4]}$ It is a stumbling block for the development of modern industry and the progress of human society. ${ }^{[5,6]}$ The economic losses caused by corrosion all over the world are impossible to evaluate. Therefore, it is of great significance to exploit some reliable and effective anticorrosion strategies. ${ }^{[7,8]}$ The organic coating located on metals plays a key role to inhibit metals' corrosion from water, oxygen, ions, aggressive medium via shielding, corrosion passivation, and electrochemical protection. ${ }^{[9-11]}$ In addition, organic coatings are easy to adhere onto metals via simple methods, and thus attract great attentions. ${ }^{[12]}$ For the organic coatings, polydimethylsiloxane (PDMS) with Si-O$\mathrm{Si}$ bond as main chain and $-\mathrm{CH}_{3}$ as side chains, endowing the high bond energy and hydrophobic group, exhibits outstanding heat resistance, flexibility, and hydrophobicity. ${ }^{[1,13]}$ PDMS has been used in various fields such as left-handed or double negative materials, ${ }^{[14]}$ cell sheet engineering, ${ }^{[15]}$ artificial skin, ${ }^{[16]}$ flexible device and humidity sensor, ${ }^{[17]}$ and corrosion resistance. ${ }^{[18,19]}$ 
However, pure PDMS coatings are easy to encounter electrolysis, even experience degradation due to the penetration of water and electrolyte through existing microspores and microcracks from its structure in the longterm usage. ${ }^{[1,10]}$ Therefore, it is necessary to modify PDMS or prepare PDMS composite coating to meet the actual application requirement. As the development of nanoscience and nanotechnology, the preparative technique and applications fields ${ }^{[20-22]}$ of nanoparticles have achieved remarkable progress. Nanoparticles are deemed as preferred material to enhance the anticorrosion performance of organic coatings. ${ }^{[10,23]}$ The modified silicon nitride with silane (KH570) maintained the corrosion resistance at $5.7 \times 10^{10} \Omega$ $\mathrm{cm}^{2}$ and adhesion strength at $7.6 \mathrm{MPa}$ after the immersion for 2400 and $1200 \mathrm{~h}$, respectively. ${ }^{[23]}$ The silica-graphene oxide $\left(\mathrm{SiO}_{2}-\mathrm{GO}\right)$ nanohybrids were introduced to epoxy resin coating, which obviously decreased the cathodic delamination rate to enhance the obstruction and to improve anticorrosion properties of the organic coating. ${ }^{[24]}$

Graphene is a typical two-dimensional nanomaterial due to its excellent electrical, mechanical and thermal properties is promising in various fields. ${ }^{[25,26]}$ Its own sheet structure can inhibit the infiltration, penetration and diffusion of corrosion media into organic coating to provide excellent shielding

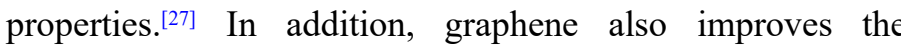
flexibility, hardness and impact resistance of the coatings. ${ }^{[28]}$ For example, Cui et al. ${ }^{[29]}$ reported that the modified graphene nanosheets with molecular-sized polydimethylsiloxane (PDMS-GNSs) exhibited a significantly enhanced dispersibility in common paint solvents and the ability to reinforce the anticorrosion performance of epoxy coating.

Alumina has been applied to enhance thermal conductivity, dielectric properties, dielectric properties, and mechanical properties of polymers due to their excellent physicochemical properties. ${ }^{[30,31]}$ At the same time, alumina is also the barrier materials to retard water vapor permeation and exhibits outstanding anticorrosion properties as a protective coating. ${ }^{[32,33]}$ In general, graphene and alumina are simultaneously dispersed in a PDMS matrix to obtain homogeneous coatings to enhance the corrosion-resistant. ${ }^{[34}$ 36]

In addition, the multilayer composites arouse great interests because they favor to prepare materials with tunable properties such as mechanical properties, ${ }^{[37]}$ permittivity, ${ }^{[38]}$ anticorrosion, ${ }^{[39]}$ and microwave absorbing ${ }^{[40]}$ according to the synergistic effects of nanofillers and interaction among adjacent layers. Therefore, in our work, the alternating multilayer PDMS nanocomposite coatings were firstly prepared using graphene and alumina via the stepwise coating method. Their adhesion, mechanical, and anticorrosion properties were characterized. Multifunctional PDMS composite coatings maintained the advantages of original components and engendered synergistic effect to overcome the inherent weak adhesion of pure PDMS. The anticorrosion property for these unique PDMS composite coating was improved as well.

\section{Experimental Section}

\subsection{Materials}

Hydroxy-terminated PDMS (750 $\mathrm{mPa} \cdot \mathrm{s})$ was purchased from Jiangxi Xinghuo Organic Silicone Plant. Graphite powders were purchased from Suzhou Carbonium Graphite Technology Co., Ltd. $\alpha$-alumina were purchased from Zhenjiang Tianyuan Warwick Nuclear New Materials Co., Ltd. $\mathrm{K}_{2} \mathrm{MnO}_{4}, \mathrm{H}_{2} \mathrm{SO}_{4}, \mathrm{H}_{2} \mathrm{O}_{2}, \mathrm{HNO}_{3}, \mathrm{NaNO}_{3}, \mathrm{NaCl}, \mathrm{P}_{2} \mathrm{O}_{5}$, $\mathrm{K}_{2} \mathrm{~S}_{2} \mathrm{O}_{8}$, and anhydrous ethanol were purchased from Sinopharm Group Co., Ltd. Ketone oxime silicone as crossing-link agent and dibutyltin dilaurate as catalyzer were purchased from Hubei Bluesky New Materials INC. The reduced graphene oxide ( $\mathrm{rGO}$ ) was prepared via the modified Hummers method. ${ }^{[41,42]}$

\subsection{Preparation of PDMS coating precursor}

Appropriate rGO, 10 g PDMS, ketoxime silane curing agent and dibutyltin dilaurate accelerator were added and stirred completely at room temperature with a high-speed dispersing machine to obtain uniform mixture. It was then put in the vacuum drying oven to eliminate the bubbles. The same method was used to obtain $\mathrm{Al}_{2} \mathrm{O}_{3}$ / PDMS coating precursor through $0.2 \mathrm{~g}$ alumina instead of graphene.

\subsection{Preparation of PDMS nanocomposite coating}

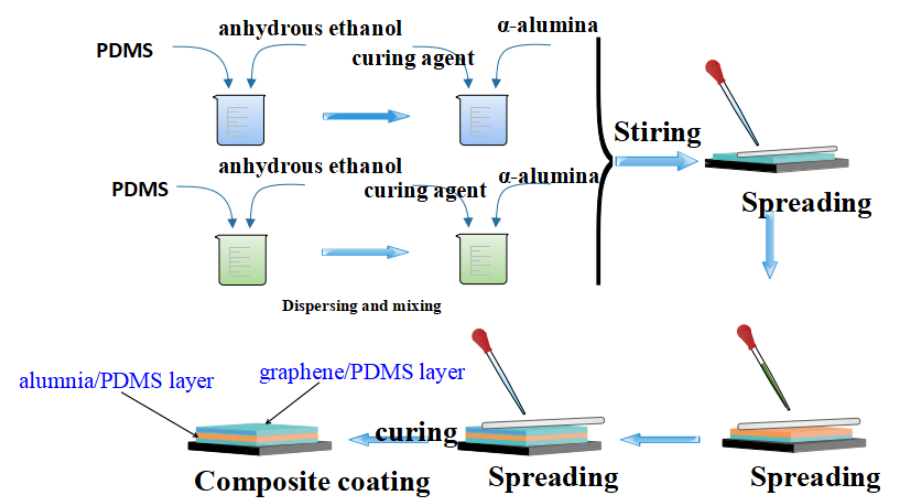

Fig. 1 the preparation scheme of alternating multilayer polydime thylsiloxane composite coating.

The metal sheet (Q235 stainless steel), the composition of which is iron and stannum, was polished with 1200\# sandpaper, the surface oxides were rinsed with anhydrous ethanol, and naturally air dried. Appropriate amount of graphene/PDMS coating precursor of which is made by the dimethyl siloxane and curing agent at the ration of 10:1.2, was evenly spread on the stainless-steel substrate. The same amount of $\mathrm{Al}_{2} \mathrm{O}_{3} / \mathrm{PDMS}$ precursor was evenly spread on the coating surface in turn. Two coating precursors were alternating used to obtain three-layer coating (1 layer of alumina coating, 2 layers of graphene coating), then it was cured for $24 \mathrm{~h}$ at room temperature, using water vapor as one of the 
curing agent to obtain alternating multilayer PDMS nanocomposite coating (see Fig. 1, the blue coating on the iron plate is PDMS mixed with graphene, and the orange coating is PDMS mixed with $\alpha$-alumina).

According to the above steps, the mass fractions of graphene were $0.050 \%, 0.1 \%, 0.3 \%, 0.5 \%$ and $1 \%$ of the PDMS, respectively. The alumina was maintained at $0.2 \%$ of the PDMS in all composite coatings. The samples were named as (1) pure PDMS, (2) $0.05 \mathrm{rGO} / 0.2 \mathrm{Al}_{2} \mathrm{O}_{3} / \mathrm{PDMS}$, (3) $0.1 \mathrm{rGO} / 0.2 \mathrm{Al}_{2} \mathrm{O}_{3} / \mathrm{PDMS}$, (4) $0.3 \mathrm{rGO} / 0.2 \mathrm{Al}_{2} \mathrm{O}_{3} / \mathrm{PDMS}$, (5) $0.5 \mathrm{rGO} / 0.2 \mathrm{Al}_{2} \mathrm{O}_{3} / \mathrm{PDMS}$, (6) $1 \mathrm{rGO} / 0.2 \mathrm{Al}_{2} \mathrm{O}_{3} / \mathrm{PDMS}$, respectively. The thickness of nanocomposite coating was about $120 \mu \mathrm{m}$

\subsection{Characterizations}

Table 1. The mechanical properties of PDMS composite coating.

\begin{tabular}{|c|c|c|c|}
\hline Samples & $\begin{array}{l}\text { Pencil } \\
\text { hardness } \\
\text { (degree) }\end{array}$ & $\begin{array}{l}\text { Adhesion } \\
\text { (degree) }\end{array}$ & Flexibility \\
\hline Pure PDMS & $6 \mathrm{~B}$ & 4 & $\begin{array}{c}\text { no whitening, } \\
\text { crack and peeling }\end{array}$ \\
\hline $\begin{array}{c}0.05 \mathrm{rGO} / 0.2 \mathrm{Al}_{2} \\
\mathrm{O}_{3} / \mathrm{PDMS}\end{array}$ & $6 \mathrm{~B}$ & 2 & $\begin{array}{l}\text { no whitening, } \\
\text { crack and peeling }\end{array}$ \\
\hline $\begin{array}{c}0.1 \mathrm{rGO} / 0.2 \mathrm{Al}_{2} \\
\mathrm{O}_{3} / \mathrm{PDMS}\end{array}$ & $6 \mathrm{~B}$ & 2 & $\begin{array}{c}\text { no whitening, } \\
\text { crack and peeling }\end{array}$ \\
\hline $\begin{array}{c}0.3 \mathrm{rGO} / 0.2 \mathrm{Al}_{2} \\
\mathrm{O}_{3} / \mathrm{PDMS}\end{array}$ & $6 \mathrm{~B}$ & 1 & $\begin{array}{c}\text { no whitening, } \\
\text { crack and peeling }\end{array}$ \\
\hline $\begin{array}{c}0.5 \mathrm{rGO} / 0.2 \mathrm{Al}_{2} \\
\mathrm{O}_{3} / \mathrm{PDMS}\end{array}$ & $6 \mathrm{~B}$ & 1 & $\begin{array}{l}\text { no whitening, } \\
\text { crack and peeling }\end{array}$ \\
\hline
\end{tabular}

The micromorphology of rGO and sectional images of nanocomposite coating were observed with a field emission scanning electron microscope (FESEM, JSM-6480, JEOL). The Raman spectra of rGO were executed using a Renishaw confocal Raman microprobe (Raman, InVia, Renishaw) at $100-2000 \mathrm{~cm}^{-1}$ of working range. The functional groups of rGO were identified by the Fourier transform infrared spectroscopy (FTIR, Cary, NYSE: A) via $\mathrm{KBr}$ pellet. The Xray diffractometer (XRD, XRD-6000, SHIMADZU) was used to identify the crystal structures of $\mathrm{rGO}$, the scan range was from $10^{\circ}$ to $80^{\circ}$.

The pencil hardness of coatings was run according at GB/T 6739-1986. The coating sample was immobilized on the pencil hardness tester. The pencil was sharpened and placed on the sandpaper to resurface the nib. The pencil was hold firmly in the pencil holder at a $45^{\circ}$ angle to the coating surface allowing the pencil to drop softly. Repeat the tests with different pencils to discern the highest level of pencil hardness whose pencil scratch the coating. The cross- cutting test was used to evaluate the adhesion of coatings according to GB/T 9286-88. Draw 6 parallel cutting marks (length:10 20mm, spacing: $1 \mathrm{~mm}$ ) on the coating with the blade, and then cut another 6 cutting marks vertically to form a small grid. The cutting marks should penetrate the coating to the substrate. Using a soft brush to brush the coating along the two diagonal lines on the grid and remove the chips and the peeling coating. Adhesion level was evaluated according the coating peeling area. Coating flexibility is executed according to GB/T 1731-1993. The sample is bent $180^{\circ}$ around the axis rob within $2-3 \mathrm{~s}$. The bending area don't occur whitening, cracking or peeling meaning the excellent flexibility.

The corrosion resistance of the composite coating was characterized using an electrochemical workstation (VersaSTAT 3-400, Ametek, USA) in $3.5 \% \mathrm{NaCl}$ solution based on three-electrode test system. The platinum electrode was used as the counter electrode, the saturated calomel electrode was used as the reference electrode, and the sample was used as the working electrode. The corrosion resistance of the composite coating was characterized by AC impedance spectroscopy and polarization curves. The $\mathrm{AC}$ impedance spectrum was measured from $0.1 \mathrm{~Hz}$ to $100 \mathrm{kHz}$ with a 10 $\mathrm{mV}$ of amplitude. The polarization curve scan rate was 1 $\mathrm{mv} / \mathrm{s}$ and the scan voltage was from -1 to $6.5 \mathrm{~V}$, and all tests were performed at ambient temperature.

\section{Results and Discussion}

The micromorphology images of GO and rGO were given as Fig. 2. It can be seen clearly that the GO is very flat and smooth three-dimensional sheet. The edge of the GO sheet is slightly folded (Fig. 2(a)). The rGO has a similar folded sheet structure (Fig.2(b)). However, the rGO displays the smaller and more wave-liked wrinkles than GO due to the transformation from $\mathrm{sp}^{3}$ to $\mathrm{sp}^{2}$ hybrid orbital in the reduction of graphene oxide. In addition, the samples were further tested via FTIR, Raman spectra, XRD (see support information) These show tat the rGO have a lower oxygen content and a higher regularity than GO.

The mechanical properties can be found in Table 1. The five samples were scratched by the $6 \mathrm{~B}$ pencil. The pencil hardness is divided into 13 grades. It decreases in turn from the hardest $6 \mathrm{H}$ to $6 \mathrm{~B}$. $6 \mathrm{~B}$ means that the five samples have the lowest hardness. That is to say, the addition of graphene and alumina does not have a significant effect to increase the hardness of PDMS. However, this does not mean that the experiment failed. On the contrary, the silicone rubber with good flexibility and high elasticity, extremely low hardness makes it an irreplaceable role in making protective coatings especially for soft devices. 

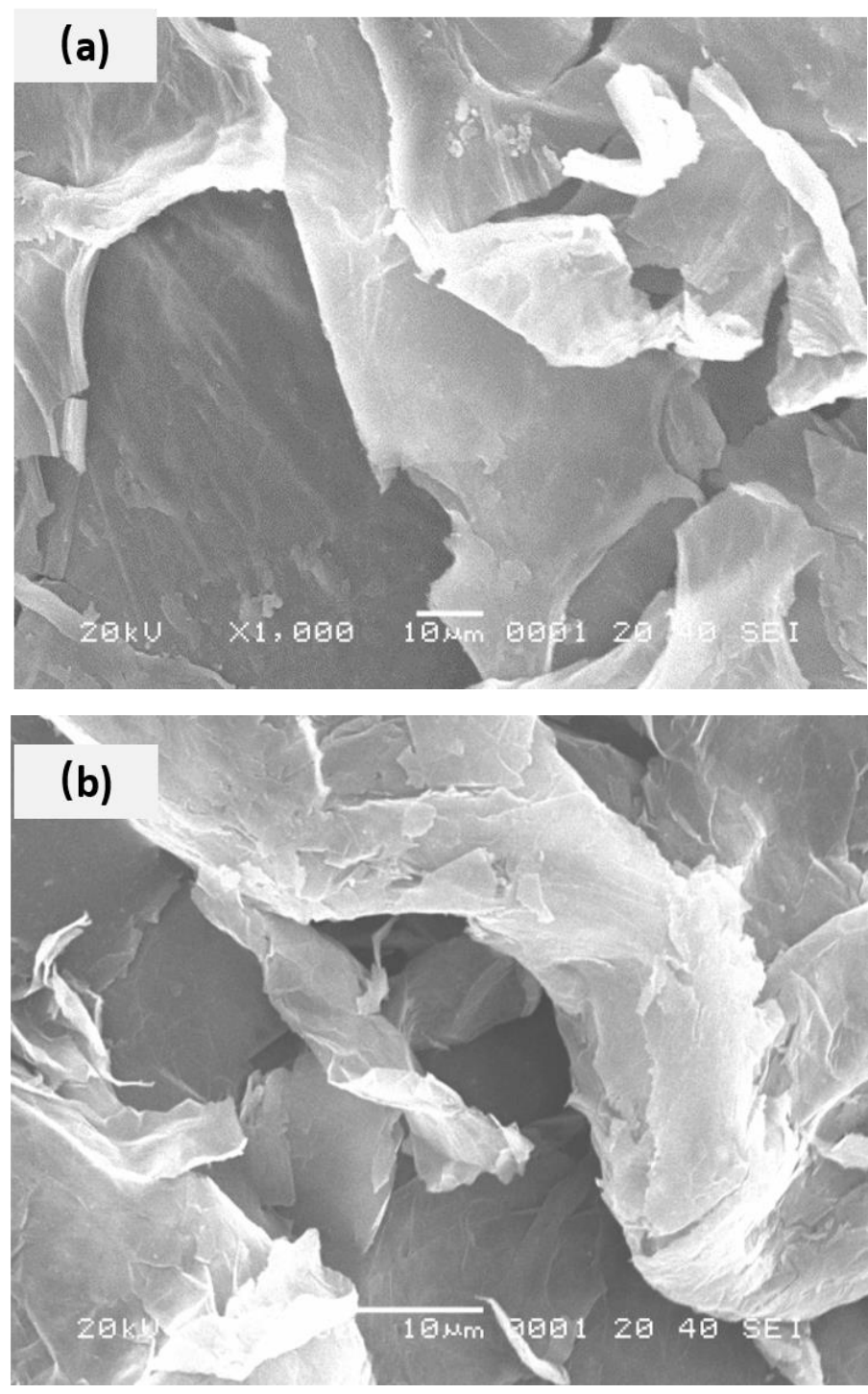

Fig. 2 SEM images of (a) graphene oxide and (b) reduced graphene oxide.

Cross-cutting is one of the easiest and most effective ways to test coating adhesion. According to the evaluation criteria, the incision of pure PDMS coating is incomplete and large-area peeling occurs. The adhesion is only 4 grades since the peeling debris area is more than $35 \%$ (Table 1 ). When the content of graphene is $0.05 \%$ and $0.1 \%$ with $0.2 \%$ alumina, the falling area is greatly decreased, which is not more than $15 \%$, so the adhesion reaches 2 levels. When the content of graphene is $0.3 \%$ and $0.5 \%$, it is almost difficult to find debris except for the negligible edge slit, and the affected area does not exceed $5 \%$, so the adhesion of composite coating can be up to level 1 . That is to say, the addition of flexible graphene can significantly improve the mechanical properties of the composite coating, and the reliable adhesion is the primary prerequisite for the coating to have good corrosion resistance.
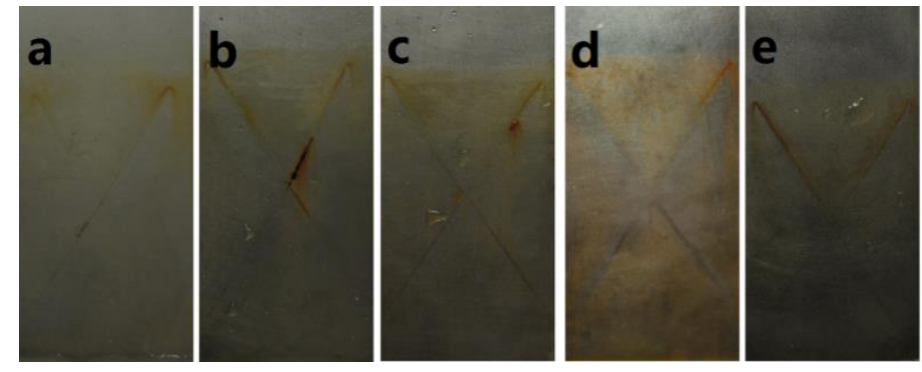

10 days
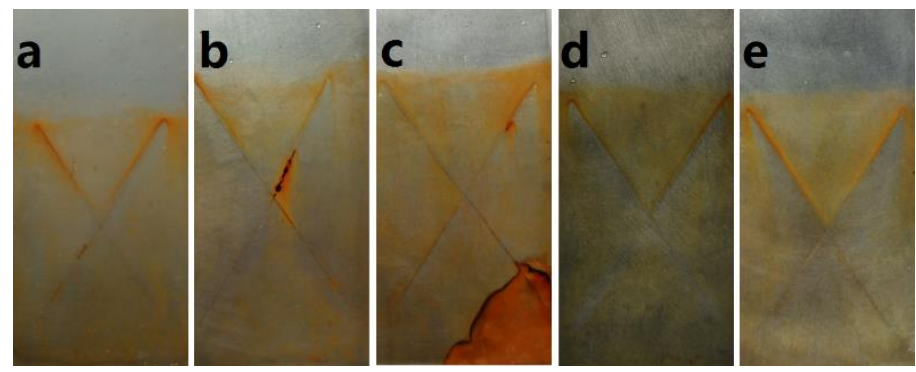

15 days
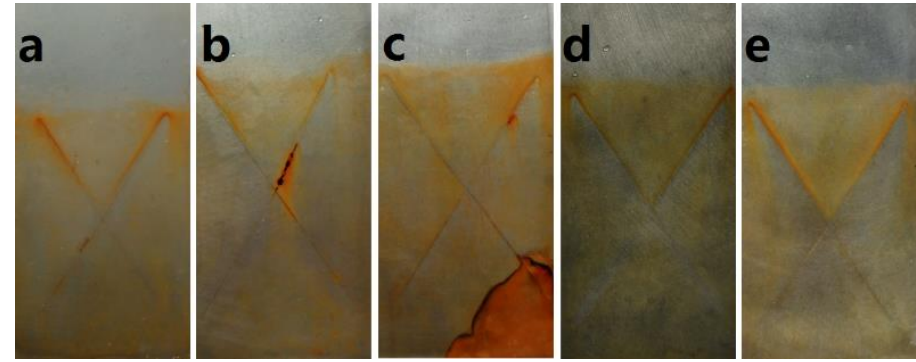

30 days

Fig. 3 The digital images of composite coating immersed in a 5 wt $\% \mathrm{NaCl}$ solution for 10, 20 and 30 days, and a, b, c, d and e represent pure PDMS, 0.05rGO/0.2 $\mathrm{Al}_{2} \mathrm{O}_{3} / \mathrm{PDMS}, 0.1 \mathrm{rGO} / 0.2$ $\mathrm{Al}_{2} \mathrm{O}_{3} / \mathrm{PDMS}, \quad 0.3 \mathrm{rGO} / 0.2 \quad \mathrm{Al}_{2} \mathrm{O}_{3} / \mathrm{PDMS}$, and $0.5 \mathrm{rGO} / 0.2$ $\mathrm{Al}_{2} \mathrm{O}_{3} / \mathrm{PDMS}$ composite coating, respectively.

Coating is easy to crack or even be detached from the surface due to the weak flexibility under the external force or environmental factors in service. Therefore, it is necessary to test flexibility in order to evaluate the degree of crack resistance and detach resistance. There is no whitening, cracking and spalling after the samples were bent, indicating that the PDMS composite coating has excellent flexibility (Table 1). The main chain of PDMS matrix is all composed of single bonds, each of which can be rotated internally, so it endows the outstanding flexibility of composite coating.

In order to evaluate the anti-corrosion effect of PDMS composite coating in practical applications, the coating was subjected to immersion test in 5\% salty solution. The coatings were edge-sealed, and then the coatings loaded on steel substrate were cross-cut. The sample was immersed in the $\mathrm{NaCl}$ solution, and it was taken out to observe at intervals. Fig. 3 is the digital images of the coatings immersed in the $\mathrm{NaCl}$ solution for different days. When soaking for 10 
days, the $0.05 \mathrm{rGO} / 0.2 \mathrm{Al}_{2} \mathrm{O}_{3} / \mathrm{PDMS}$ coating appears first rust (Fig. $3 b_{1}$ ). This is because when the content of fillers is too low, there is not enough physical barrier in the coating, and even its excellent electrical conductivity would promote the corrosion due to the cross-shaped damage. With the increase of rGO content, the anti-corrosion performance of the coating is improved, demonstrating a good protection on the substrate due to enough physical barrier. For the immersion of 20 days, the $0.05 \mathrm{rGO} / 0.2 \mathrm{Al}_{2} \mathrm{O}_{3} / \mathrm{PDMS}$ coating begins to be detached due to the edge sealing (Fig. $3 \mathrm{c}_{2}$ ). When the immersion is executed for 30 days, the pure PDMS, $0.05 \mathrm{rGO} / 0.2 \mathrm{Al}_{2} \mathrm{O}_{3} / \mathrm{PDMS}$ and $0.1 \mathrm{rGO} / 0.2 \mathrm{Al}_{2} \mathrm{O}_{3} / \mathrm{PDMS}$ coatings appear a large range of peeling and infection, and the three sets of coatings basically failed. Fortunately, the pure PDMS, $0.3 \mathrm{rGO} / 0.2 \quad \mathrm{Al}_{2} \mathrm{O}_{3} \quad / \mathrm{PDMS} \quad$ and $0.5 \mathrm{rGO} / 0.2 \mathrm{Al}_{2} \mathrm{O}_{3} / \mathrm{PDMS}$ coatings only have some yellow rust spots. The coatings of $0.5 \mathrm{rGO} / 0.2 \mathrm{Al}_{2} \mathrm{O}_{3} / \mathrm{PDMS}$ shows the darker rust spot than $0.3 \mathrm{rGO} / 0.2 \mathrm{Al}_{2} \mathrm{O}_{3} / \mathrm{PDMS}$, indicating the more rGO is probable to agglomerate and cause some microscopic mesoporous. It indicates that the corrosive medium such as water and oxygen has passed through microscopic mesoporous to the substrate after the long immersion.
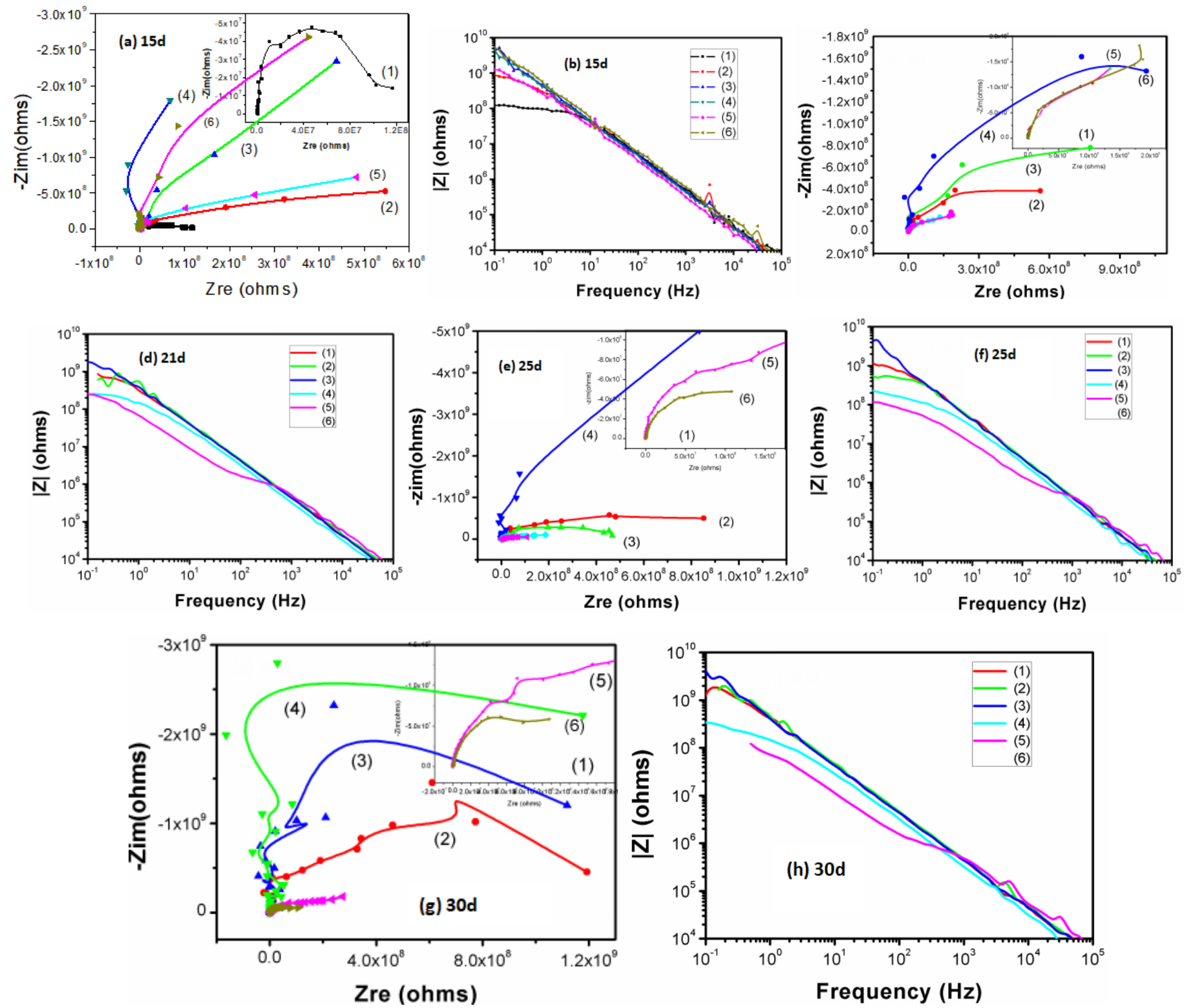

Fig. 4 Nyquist plots (a) 15d, (c) 21d, (e) 25d, (g) 30d and Bode plots (b) 15d, (d) 21d, (f) 25d, (h) 30d and $|\mathrm{Z}|(0.01 \mathrm{HZ}$ ) of different exposuretime during 30 days. Samples ((1) pure PDMS, (2) 0.05rGO/0.2 $\mathrm{Al}_{2} \mathrm{O}_{3} / \mathrm{PDMS}$, (3) 0.1 rGO/0.2 $\mathrm{Al}_{2} \mathrm{O}_{3} / \mathrm{PDMS}$, (4) $0.3 \mathrm{rGO} /$ $0.2 \mathrm{Al}_{2} \mathrm{O}_{3} / \mathrm{PDMS}$, (5) 0.5rGO/0.2 $\mathrm{Al}_{2} \mathrm{O}_{3} / \mathrm{PDMS}$, (6) $1 \mathrm{rGO} / 0.2 \mathrm{Al}_{2} \mathrm{O}_{3} / \mathrm{PDMS}$ ) were immersed in different time. 
Electrochemical impedance spectroscopy (EIS) using the small amplitude sinusoidal wave potential (or current) as a di sturbance signal is widely used[43]. The Nyquist and Bode pl ots are two different types of diagrams of the $\mathrm{AC}$ impedance spectrum. The Nyquist is especially suitable for representing the impedance of the system to deduce the circuit component $\mathrm{s}$ and simulate the equivalent circuit, which is the impedance imaginary part (-Zim) versus the real part of the impedance (Zre). The Bode is plotted according the logarithm of the imp edance modulus $(\log |Z|)$ versus the logarithm of the frequenc $\mathrm{y}(\log \mathrm{F})$. The Bode plot can well explain the corrosion resista nce of the coating especially in the low frequency region. Fi g. 4 shows the Nyquist and Bode plots of five coatings for dif ferent soaking days and $|\mathrm{Z}|(0.01 \mathrm{HZ})$ VS exposure time in diff errent time. It can be seen from the Nyquist that the capacitiv e arc resistance of composite coatings is not very obvious exc ept for the pure PDMS coating samples at the beginning of th e immersion (Fig.4a). The Bode shows that the impedance $m$ odulus of the coatings are all almost above $10^{9} \Omega \bullet \mathrm{cm} 2$ (Fig. 4
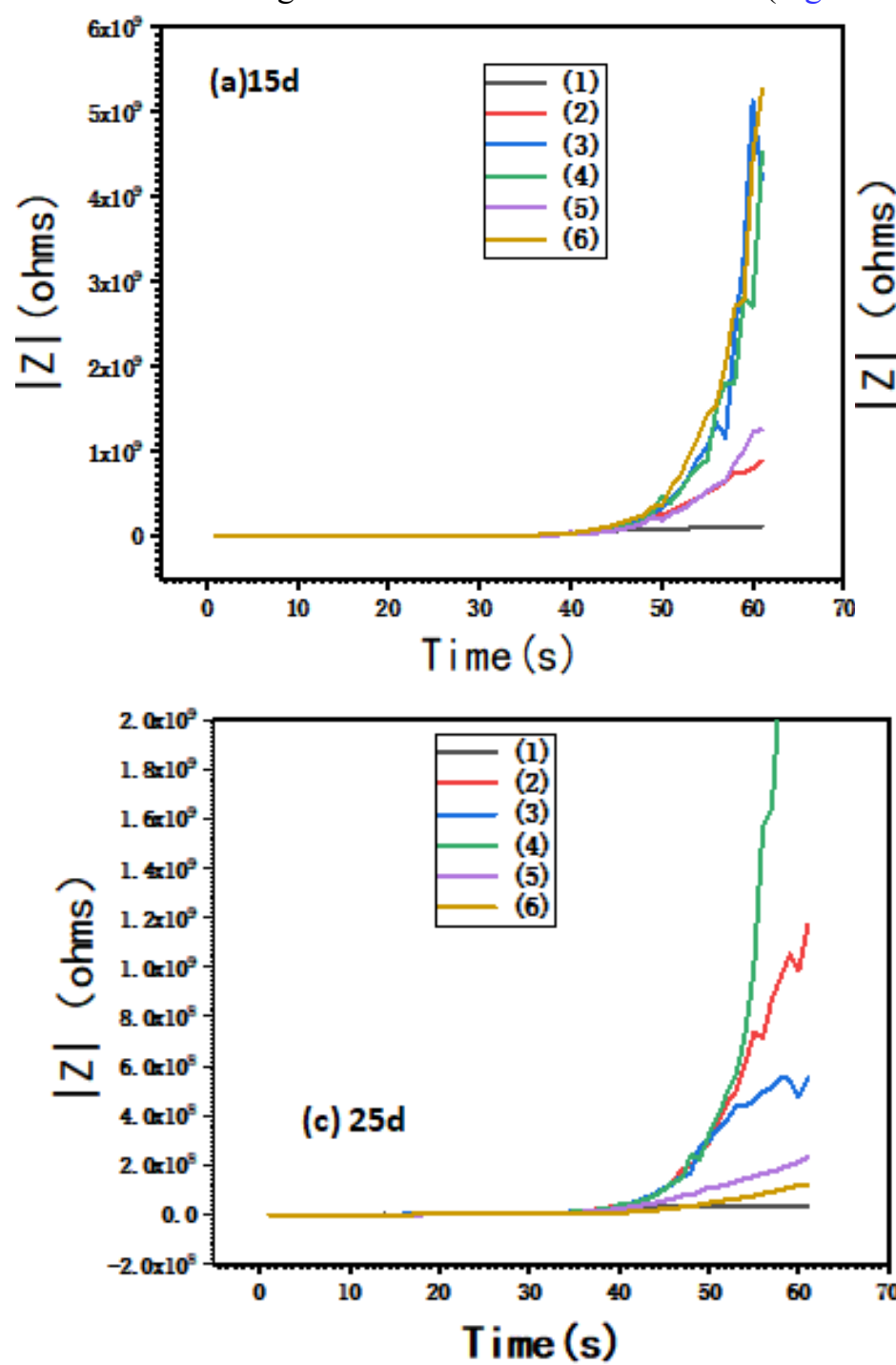

b) at the low frequency. It indicates that the coating at this ti me is equivalent to a pure capacitor, which can effectively av oid the direct contact between the metal substrate and the cor rosion solution, and has a good protection as a barrier on the substrate, thereby preventing the metal substrate from corrodi ng.

After 21 and 25 days of immersion, it can be clearly seen from the Nyquist diagram (Fig. $4 \mathrm{c}$ and e) that as the immersion time increases, the coating capacity arc begins to show a bending tendency. The Bode plot (Fig. 4d and f) displays that the impedance modulus of the $0.05 \mathrm{rGO} / 0.2 \mathrm{Al}_{2} \mathrm{O}_{3} / \mathrm{PDMS}, 0.3 \mathrm{rGO} / 0.2 \mathrm{Al}_{2} \mathrm{O}_{3} / \mathrm{PDMS}$ samples is still $10^{9} \Omega \cdot \mathrm{cm}^{2}$ and others have been greatly reduced. It shows that the transfer resistance of the coating is reduced, the corrosion resistance begins to decrease, and the corrosive medium begins to seep to the surface of the metal sheet through the protective coating. But due to the large impedance, it still has an effective corrosion resistance effect.
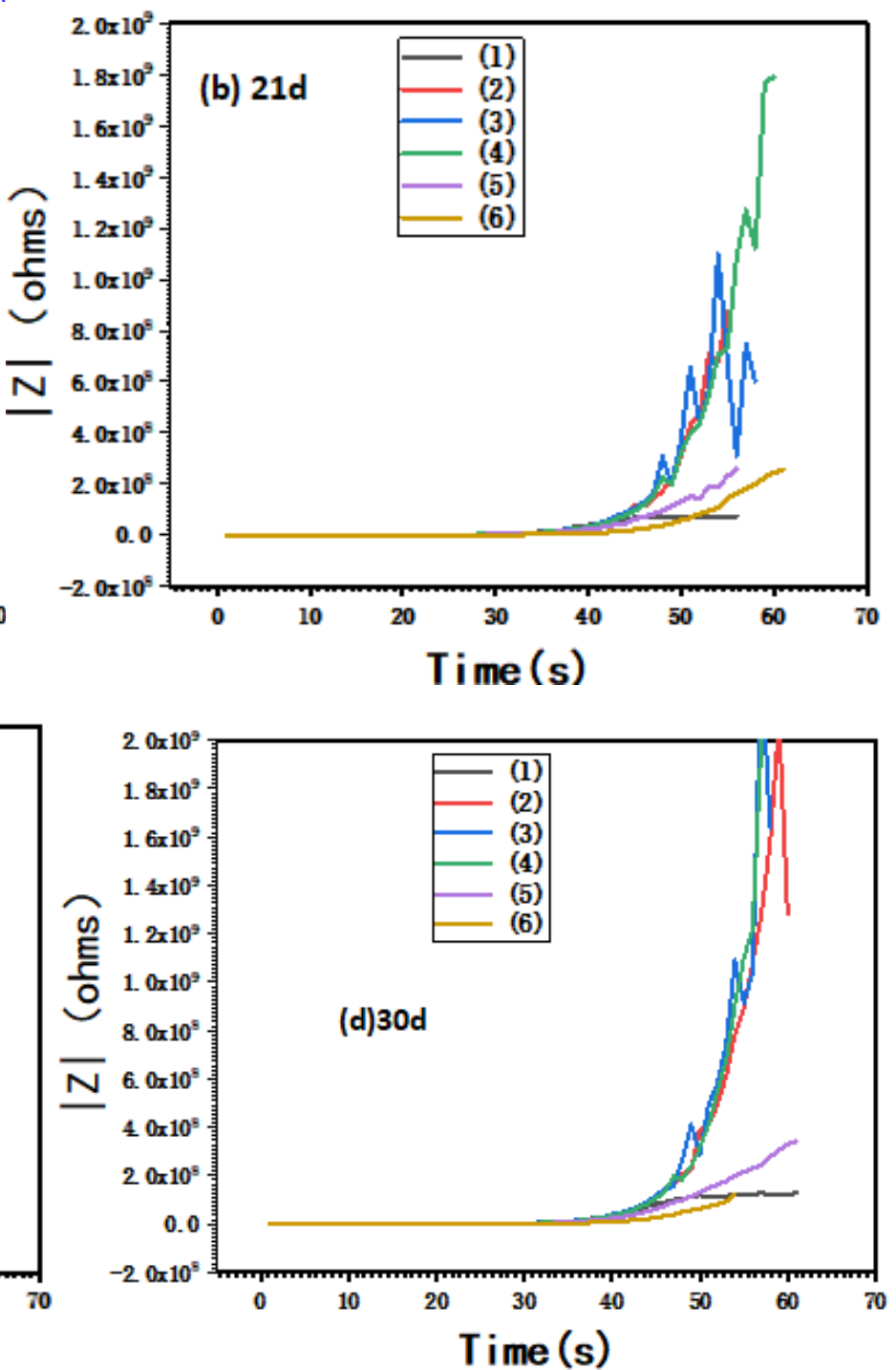

Fig. $5|\mathrm{Z}|(0.01 \mathrm{HZ})$ of different exposure time during 30 days. 
Table 2. Impedance modulus $|\mathrm{Z}|\left(\Omega \bullet \mathrm{cm}^{2}\right)$ of samples soaked in different low frequency zones.

\begin{tabular}{|c|c|c|c|c|c|c|}
\hline \multirow{2}{*}{ Soaking days } & \multicolumn{6}{|c|}{ Samples } \\
\hline & (1) & (2) & (3) & (4) & (5) & (6) \\
\hline $15 \mathrm{~d}$ & $1.23 \times 10^{8}$ & $8.9 \times 10^{8}$ & $4.23 \times 10^{9}$ & $4.19 \times 10^{9}$ & $1.25 \times 10^{9}$ & $5.27 \times 10^{9}$ \\
\hline $21 d$ & $7.29 \times 10^{7}$ & $8.74 \times 10^{8}$ & $8.37 \times 10^{8}$ & $1.79 \times 10^{9}$ & $2.50 \times 10^{8}$ & $2.43 \times 10^{8}$ \\
\hline $25 \mathrm{~d}$ & $3.12 \times 10^{7}$ & $1.17 \times 10^{9}$ & $5.53 \times 10^{8}$ & $4.19 \times 10^{9}$ & $2.31 \times 10^{8}$ & $1.15 \times 10^{8}$ \\
\hline $30 \mathrm{~d}$ & $1.26 \times 10^{8}$ & $1.27 \times 10^{9}$ & $1.64 \times 10^{9}$ & $4.16 \times 10^{9}$ & $3.41 \times 10^{8}$ & $1.22 \times 10^{8}$ \\
\hline
\end{tabular}

Notes: (1) pure PDMS, (2) 0.05rGO/0.2 $\mathrm{Al}_{2} \mathrm{O}_{3} / \mathrm{PDMS}$, (3) 0.1rGO/0.2 $\mathrm{Al}_{2} \mathrm{O}_{3} / \mathrm{PDMS}$, (4) 0.3rGO/0.2 $\mathrm{Al}_{2} \mathrm{O}_{3} / \mathrm{PDMS}$, (5) 0.5rGO/0.2 Al $2 \mathrm{O}_{3} / \mathrm{PDMS}$, (6) $1 \mathrm{rGO} / 0.2 \mathrm{Al}_{2} \mathrm{O}_{3} / \mathrm{PDMS}$.

When the immersion reaches up to 30 days, the capacitive anti-arc of the Nyquist (Fig. 4g) occur a very obvious bending curvature, which is approximately a semicircle, and the diameter of the capacitive anti-arc decrease continuously. The impedance modulus of Bode (Fig. 4h) also displays the similar trend to decrease. Fig. 5 are curves of $|\mathrm{Z}|(0.01 \mathrm{HZ})$ of different exposure time during 30 days. It means that with the immersion time prolonged, the coating is gradually destroyed due to the permeation of corrosive medium. The reaction at this stage is mainly controlled by the corrosion diffusion rate. The addition of graphene and alumina endows the coating excellent shielding and isolation for the metal substrate. Therefore, the coatings exhibit the bigger diameter of the capacitive anti-arc and impedance than the pure PDMS coating. However, the agglomeration of graphene when the content is more than $0.3 \%$ which may lead to micropores, which provided a channel for the diffusion of the corrosive medium such as water and electrolyte to penetration. The coating with $0.3 \%$ has the best anti-corrosion ability, which is consistent with the previous immersion test results (Fig. 3).

In order to more intuitively understand the corrosion in the different phases of composite coating immersed in salt water, the impedance modulus $(\log |\mathrm{Z}|)$ of all samples (Table. 2) and Nyquist of $1 \mathrm{rGO} / 0.2 \mathrm{Al}_{2} \mathrm{O}_{3} / \mathrm{PDMS}$ composite coating was separately plotted (Fig. 6). It can be more clearly seen from Fig. 6 that the radius of curvature is gradually reduced, indicating that the transfer resistance of the coating is reduced. Table. 1 displayed $1.22 \times 10^{8} \Omega \cdot \mathrm{cm}^{2}$ of impedance modulus for the coating immersed for 30 days which is reduced by about 1 orders of magnitude compared the samples immersed for $15 \mathrm{~d}$. Although there is some fluctuation for the impedance modulus, the overall trend is dropping down, indicating that the corrosion resistance ability of composite coating decline in the process of immersion.
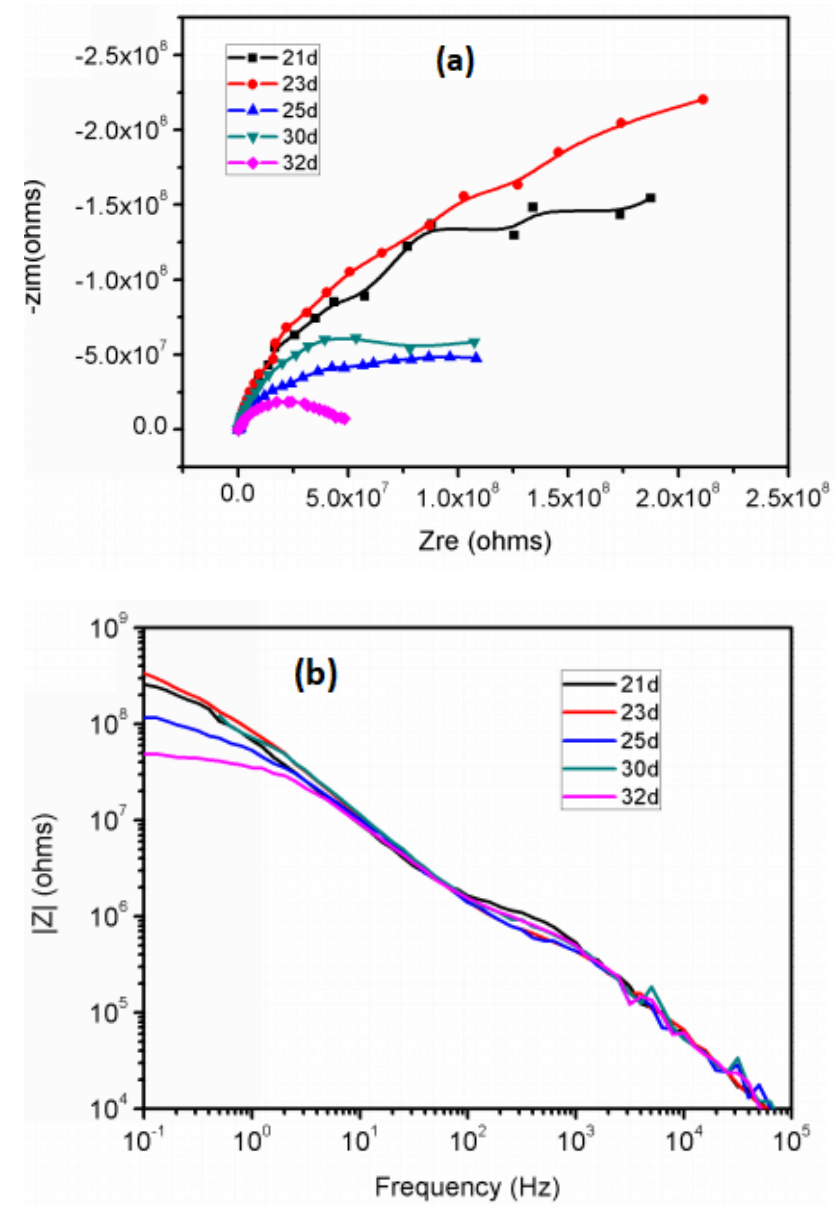

Fig. 6 Nyquist plots (a) and Bode plots (b) for different days of $1 \mathrm{rGO} / 0.2 \mathrm{Al}_{2} \mathrm{O}_{3} / \mathrm{PDMS}$.

The equivalent circuit also is fitted according the electrochemical impedance curves to explain their electrochemical reaction process (Fig. 7), where Rs is the solution resistance, $\mathrm{Qc}$ is the coating capacitance, $\mathrm{Rc}$ is the coating resistance, Qdl is the electric double layer capacitance, $\mathrm{Rt}$ is the electron migration resistance, and $\mathrm{Zw}$ is the Warburg impedance. The equivalent circuit is different at different soaking stages meaning the corrosion behavior is 
different. At the initial stage of coating immersion, the corrosive mediums such as water, air and electrolyte are isolated by the coating, and the coating play the role of excellent protection. the coating capacitance is in parallel with the coating resistance in the equivalent circuit (Fig. 8a), where $\mathrm{Rs}$ is the solution resistance, Qc is the coatingcapacitance, $\mathrm{Rc}$ is the coating resistance. The coating is equivalent to a complete shielding layer with a large resistance and a small capacitance. The coating has a good protection effect, and corrosive medium such as water and oxygen cannot penetrate the coating. In the middle of immersion, the corrosive medium penetrates via the micropores and gaps in the coating to the substrate, and generates an electric double layer capacitance and induces a corrosion reaction on the surface of the substrate. At this stage, the charge transfer resistance and the electric double layer capacitance exist simultaneously, and thus they are in a parallel relationship ((Fig. 7b), where Qdl is the electric double layer capacitance, Rt is the electron migration resistance. The electrochemistry of the metal substrate is just beginning and the reaction area is small, so the coating still has protective effect. In the late stage of immersion, the corrosive medium invades the surface of the metal substrate, and deep corrosion further occurs. At this stage, the corrosion is mainly controlled by the diffusion process, so the diffusion resistance and capacitance equivalent circuit (Fig. 7c), where $\mathrm{Zw}$ is the impedance corresponding to diffusion information. The coating is basically failed to protect metal as the continual diffusion.

It can directly evaluate the corrosion resistance of the system by observing the characteristic potential value on the polarization curve (such as self-corrosion potential and current). So, the polarization curve, corrosion potential vs corrosion current and corrosion potential (Ecorr, icorr) VS exposure time are displayed in Fig. 8 and Table. 2. Figs. 8 (a) and (b) is the curve of corrosion potential (Ecorr, icorr) of different exposure time. Since the corrosion potential of the coating with $0.3 \%$ graphene is too large compared with other samples, the polarization curve is drawn separately (Fig. 8d). In the general, the smaller the corrosion current density, the lower the corrosion rate and the better the corrosion resistance. It can be seen from Figs. 8(c) and (d) that the coating with fillers display evident lower corrosion current than that of the pure PDMS coating. The corrosion current density first decreases and then increases with the increase of fillers contents. The corrosion potential has the opposite tendency and transfer from negative to positive when addition of fillers. The corrosion potential shows a trend of increasing first and then decreasing with the increase of fillers contents. This is because that the addition of a proper amount of fillers can effectively reduce the intrusion of corrosive medium due to its barrier shielding effect. However, when the fillers content is too high, agglomeration occurs in the composite coating to arise micropore, making the corrosive medium easier to penetrate. The composite coating with $0.3 \%$ graphene shows the best corrosion resistance, with the corrosion potential as high as $4.82 \mathrm{v}$, which was about 40 times higher than the pure PDMS coating.

The composite coating possesses outstanding corrosion resistance mainly due to the following two reasons (see Fig. 9): (1) Shielding effect. The compatible fillers can improve the defects of the organic coating, such as holes and dents, and make the coating more compact, avoiding the direct contact between the metal substrate and the corrosive medium. In addition, the stacking between layers of graphene form a tight physical isolation layer, which extend the penetration path of corrosion medium in coating. (2) Electrochemical protection. The conductivity of the coating ascends due to the excellent conductivity of fillers. When the corrosion occurs on the substrate, the metal Fe as anode loses electrons and generates $\mathrm{Fe}^{3+}$. The electrons quickly transfer to the surface of coating due to the high conductivity endowed by the graphene. Therefore, reduction reaction of cathode occurs on the surface of coating. The distance between the cathode and anode reaction eventually lead to the decrease of corrosion reaction rate and obviously delay the corrosion process.

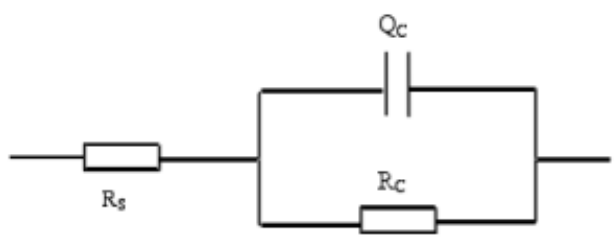

(a)

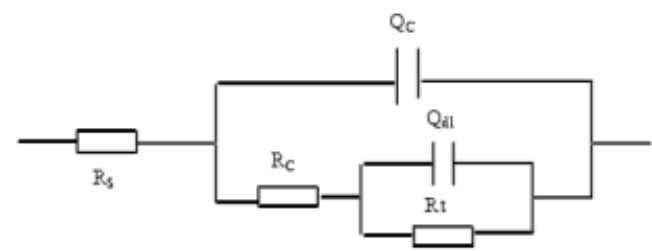

(b)

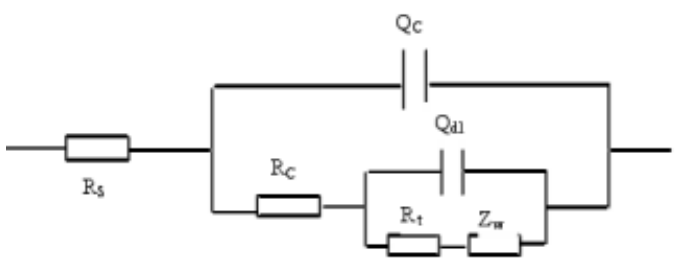

(c)

Fig. 7 Equivalent circuit diagram of the (a) initial, (b) middle and late stages of immersion of simulated coating in $3.5 \% \mathrm{NaCl}$ solution. 

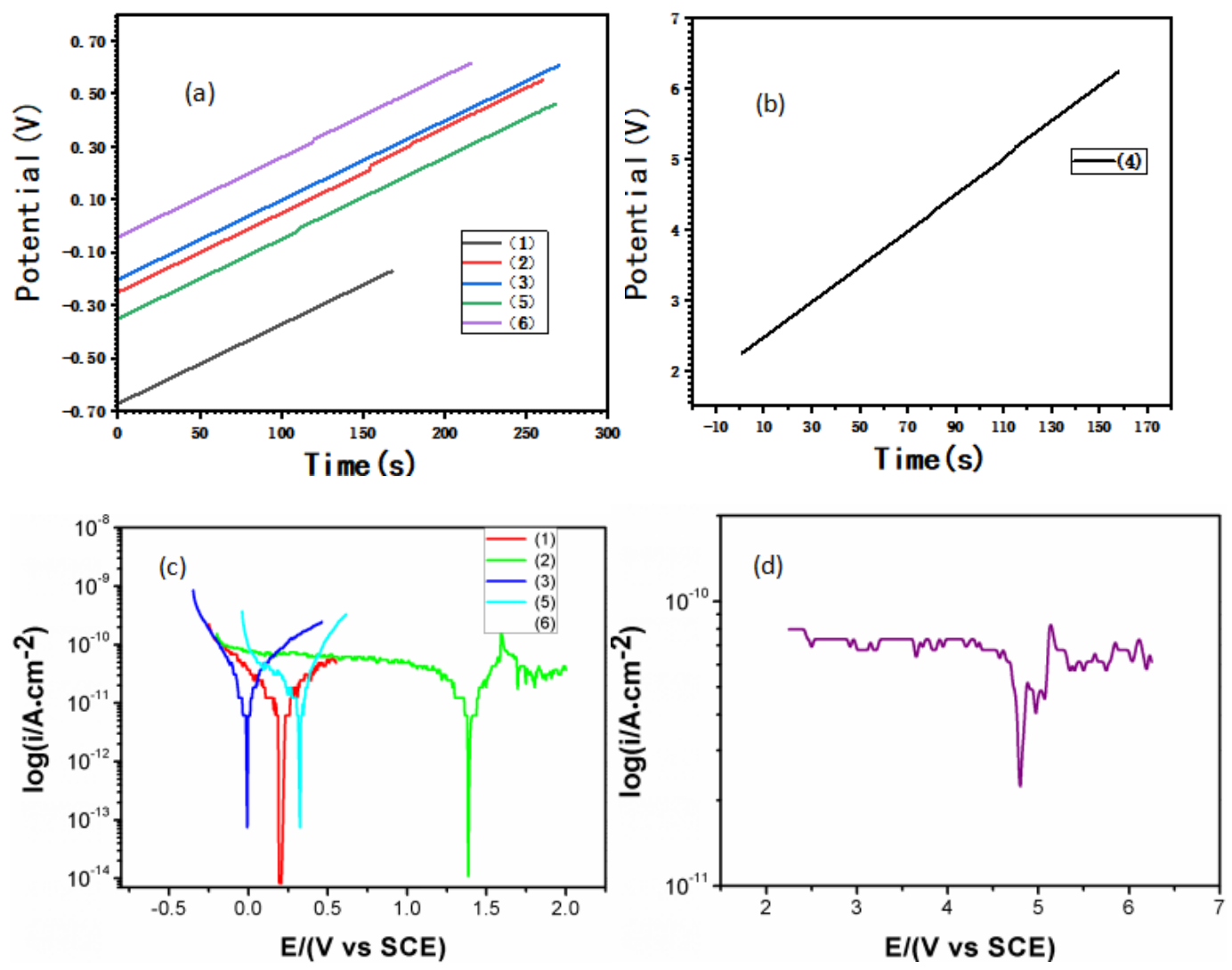

Fig 8 (a) and (b) The curve of corrosion potential (Ecorr, icorr) at different exposure time, Polarization curve (c) of the sample of (1), (2), (3), (5), (6) in a solution of $3.5 \mathrm{wt} \% \mathrm{NaCl}$, Polarization curve (d) of the sample of group (4) ((1) pure PDMS, (2) 0.05rGO/0.2 $\mathrm{Al}_{2} \mathrm{O}_{3} / \mathrm{PDMS}$, (3) 0.1 rGO/0.2 $\mathrm{Al}_{2} \mathrm{O}_{3} / \mathrm{PDMS}$, (4) 0.3rGO/0.2 $\mathrm{Al}_{2} \mathrm{O}_{3} / \mathrm{PDMS}$, (5) 0.5 $\mathrm{rGO} / 0.2 \mathrm{Al}_{2} \mathrm{O}_{3} / \mathrm{PDMS}$, (6) $1 \mathrm{rGO} / 0.2$ $\mathrm{Al}_{2} \mathrm{O}_{3} / \mathrm{PDMS}$ ).

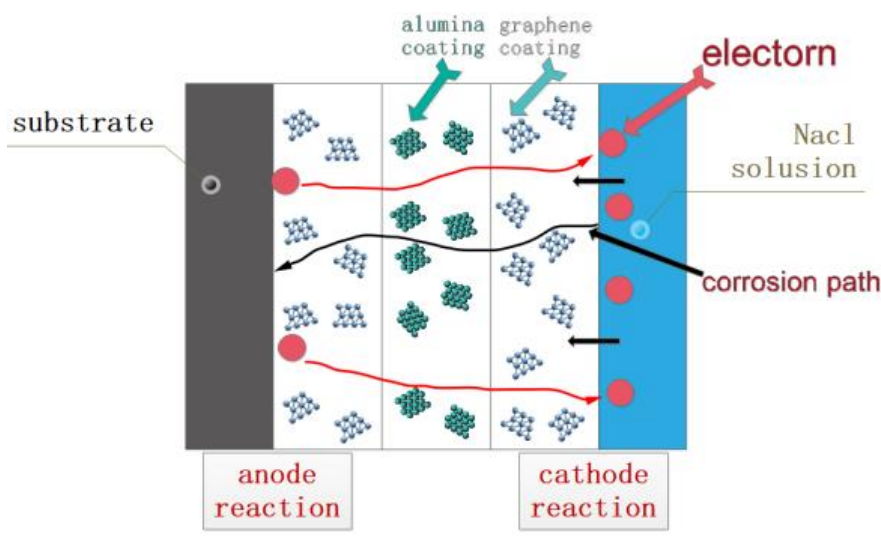

Fig. 9 The anticorrosion mechanism of alternating multilayer po lydimethylsiloxane composite coating.

\section{Conclusion}

The alternating multilayer PDMS composite coating was prepared via a stepwise coating method based on graphene and alumina used as fillers. Adhesion is greatly improved when the coating remains extreme flexibility. The fillers effectively impede the corrosion medium and immensely improve the anticorrosive performance of the composite coating. When the mass fraction of filler is $0.3 \%$, the composite coating displays the best anticorrosive property of the coating. The impedance modulus value reaches above
$10^{9} \Omega \cdot \mathrm{cm}^{2}$, and $\mathrm{E}$ corr reaches $4.82 \mathrm{v}$, which is about 40 times higher than the pure PDMS coating. So, the epoxy composites with alternating binary fillers achieve obvious anticorrosion to protect metal substrates.

\section{Acknowledgments}

This work was financially supported by National Nature Science Foundation of China (No. 51402132), Jiangsu Government Scholarship for Overseas Studies (No. JS-2016136), the Key Laboratory of High-tech Research on Marine Functional Thin Film Materials in Zhenjiang (ZHZ2019008), and Jiangsu Provincial Natural Science Foundation of China (BK20160562).

\section{Supporting information}

Not applicable.

\section{Conflict of interest}

There are no conflicts to declare.

\section{References}

[1] G. Zhu, X. Cui, Y. Zhang, S. Chen, M. Dong, H. Liu, Q. Shao, T. Ding, S. Wu, Z. Guo, Polymer, 2019, 172 , 415-422.

[2] Z. Szklarska-Smialowska, Corro. Sci., 1999, 41, 1743-1767, doi: 10.1016/j.polymer.2019.03.056.

[3] K. Gong, X. Li, H. Liu, X. Cheng, D. Sun, Q. Shao, M. Dong, C. Liu, S. Wu, T. Ding, Carbon, 2020, 156, 320-328, doi: 10.1016/j.carbon.2019.09.046. 
[4] C. M. Caldas, L.F. Calheiros, B. G. Soares, J. App. Poly. Sci., 2017, 134, 45505, doi: 10.1002/app.45505.

[5] C. Zheng, B. Yan, K. Zhang, G. Yi, Inter. J. Mine., Metall., Mate., 2015, 22, 729-737, doi: 10.1007/s12613-015-1128-5.

[6] T. Yanbing, L. Zhihong, Q. Yanxin, G. Pingyi, L. Gang, W. Baigong, G. Hao, Corr. Sci. and Prote. Tech., 2018, 30, 449453, doi: 10.11903/1002.6495.2017.224.

[7] X. Lu, X. Feng, Y. Zuo, P. Zhang, C. Zheng, Prog. in Organ. Coat., 2017, 104, 188-198, doi: 10.1016/j.porgcoat.2016.11.001. [8] P. Wang, Z. Lu, D. Zhang, Corr. Sci., 2015, 93, 159-166, doi: 10.1016/j.corsci.2015.01.015.

[9] C. Yang, H. Wei, L. Guan, J. Guo, Y. Wang, X. Yan, X. Zhang, S. Wei, Z. Guo, J. of Mater. Chem. A, 2015, 3, 14929 14941, doi: 10.1039/C5TA02707A.

[10] X. Cui, G. Zhu, Y. Pan, Q. Shao, M. Dong, Y. Zhang, Z. Guo, Polymer, 2018, 138, 203-210, doi: 10.1016/j.polymer.2018.01.063.

[11] X. Gong, Y. Liu, S. Wu, D. Ding, H. Wei, Z. Guo, J. of App. Poly. Sci., 2015, 132, 41648, doi: 10.1002/app.41648.

[12] H. Amirbeygi, H. Khosravi, E. Tohidlou, J. of App. Poly. Sci., 2019, 136, 47410, doi: 10.1002/app.47410.

[13] Z. Zhang, J. Zhang, S. Li, J. Liu, M. Dong, Y. Li, N. Lu, S. Lei, J. Tang, J. Fan, Comp. Part B: Engg., 2019, 176, 107338, doi: 10.1016/j.compositesb.2019.107338.

[14] K. Sun, P. Xie, Z. Wang, T. Su, Q. Shao, J. Ryu, X. Zhang, J. Guo, A. Shankar, J. Li, Polymer, 2017, 125, 50-57, doi: 10.1016/j.polymer.2017.07.083.

[15] D. Sharma, W. Jia, F. Long, S. Pati, Q. Chen, Y. Qyang, B. Lee, C.K. Choi, F. Zhao, Bioact. mater, 2019, 4, 142-150, doi: 10.1016/j.bioactmat.2019.02.002.

[16] G. Shi, Y. Wang, S. Derakhshanfar, K. Xu, W. Zhong, G. Luo, T. Liu, Y. Wang, J. Wu, M. Xing, Mater. sci. \& engg. C, 2019, 100, 915-927, doi: 10.1016/j.msec.2019.03.058.

[17] Y. Wu, Q. Huang, J. Nie, J. Liang, N. Joshi, T. Hayasaka, S. Zhao, M. Zhang, X. Wang, L. Lin, J. of Nanosci. and Nanotech., 2019, 19, 5310-5316, doi: 10.1166/jnn.2019.16821.

[18] D. Li, H.-Y. Wang, Y. Liu, D.-S. Wei, Z.-X. Zhao, Chem. Engg J., 2019, 367, 169-179, doi: 10.1016/j.cej.2019.02.093.

[19] B. Gonçalves, L. Silva, T. Souza, V. Castro, G. Silva, B. Silva, K. Krambrock, R.B. Soares, V.F. Lins, M. Houmard, J. Mater. Resea., 2019, 34, 3918-3930, doi: 10.1557/jmr.2019.342. [20] J. Zhang, Z. Zhang, Y. Jiao, H. Yang, Y. Li, J. Zhang, P. Gao, J. Pow. Sour., 2019, 419, 99-105, doi: 10.1016/j.jpowsour.2019.02.059.

[21] M. Liu, Q. Meng, Z. Yang, X. Zhao, T. Liu, Chem. Comm., 2018, 54, 5090-5093, doi: 10.1039/c8cc01889h.

[22] S. Liu, L. Liang, L. Meng, X. Tian, Z. Zhang, Y. Yu, Z. Lan, J. Wu, J. Zhang, P. Gao, Solar RRL, 2020, 4, 1900231, doi: 10.1002/solr.201900231.

[23] Y. Zhang, M. Zhao, J. Zhang, Q. Shao, J. Li, H. Li, B. Lin, M. Yu, S. Chen, Z. Guo, J. Poly. Resea., 2018, 25, 130, doi; 10.1007/s10965-018-1518-2.

[24] B. Ramezanzadeh, Z. Haeri, M. Ramezanzadeh, Chem. Engg J., 2016, 303, 511-528, doi: 10.1016/j.cej.2016.06.028.
[25] S. Li, P. Yang, X. Liu, J. Zhang, W. Xie, C. Wang, C. Liu, Z. Guo, J. Mater. Chem A, 2019, 7, 16902-16911, doi; 10.1039/C9TA04562G.

[26] Y. Guo, X. Yang, K. Ruan, J. Kong, M. Dong, J. Zhang, J. Gu, Z. Guo, ACS Appl. Mater. interfs., 11 (2019) 25465-25473, doi: 10.1021/acsami.9b10161.

[27] Y. Jiao, J. Zhang, S. Liu, Y. Liang, S. Li, H. Zhou, J. Zhang, Sci. of Adv. Mater., 2018, 10, 1706-1713, doi: 10.1166/sam.2018.3338.

[28] J. Ding, O. U. Rahman, W. Peng, H. Dou, H. Yu, Appl. Surf. Sci., 2018, 427, 981-991, doi: 10.1016/j.apsusc.2017.08.224.

[29] W. Sun, L. Wang, Z. Yang, T. Zhu, T. Wu, C. Dong, G. Liu, Mater. Lett., 2018, 228, 52-156, doi: 10.1016/j.matlet.2018.05.105.

[30] M. Sangermano, M. Naguib, M. Messori, Macromol. Mater. and Engg., 2013, 298, 1184-1189, 10.1002/mame.201200346.

[31] J. D. Oliveira, R.C. Rocha, A. G. de Sousa Galdino, J. of Mater. Resea. and Tech., 2019, 8, 1729-1736, doi: 10.1016/j.jmrt.2018.10.016.

[32] K.L. Jarvis, P.J. Evans, G. Triani, Surf. and Coat. Techno., 2018, 337, 44-52, doi: 10.1016/j.surfcoat.2017.12.056.

[33] Q. Li, Y. Zhang, Acta Phys. Sin. 2018, 67, 067201, doi: 10.7498/aps.67.20172311.

[34] R. Qian, J. Yu, C. Wu, X. Zhai, P. Jiang, Rsc Adv., 2013, 3 17373-17379, doi: 10.1039/C3RA42104J.

[35] Y. Feng, C. He, Y. Wen, X. Zhou, X. Xie, Y. Ye, Y.-W. Mai, Compo. Sci. and Tech., 2018, 160, 42-49, doi: 10.1016/j.compscitech.2018.03.009.

[36] Z. Yu, H. Di, Y. Ma, L. Lv, Y. Pan, C. Zhang, Y. He, App. Surf. Sci., 2015, 351, 986-996, doi; 10.1016/j.apsusc.2015.06.026.

[37] Y. Wang, J. Cui, Q. Yuan, Y. Niu, Y. Bai, H. Wang, Adv. Mater., 2015, 27, 6658-6663, doi: 10.1002/adma.201503186.

[38] J. Wang, Z. Shi, F. Mao, S. Chen, X. Wang, ACS appl. Mater. Interf., 2017, 9, 1793-1800, doi: 10.1021/acsami.6b12786.

[39] J. Zhang, W. Zhang, L. Wei, L. Pu, J. Liu, H. Liu, Y. Li, J. Fan, T. Ding, Z. Guo, Macromol. Mater. and Engg., 2019, 304, 1900374, doi: 10.1002/mame.201900374.

[40] Y. Gao, X. Gao, J. Li, S. Guo, J. Appl. Poly. Sci., 2018, 135, 45846, doi: 10.1002/app.45846.

[41] W.S. Hummers Jr, R.E. Offeman, J. Ameri Chem. Soc., 1958, 80, 1339-1339, doi: 10.1021/ja01539a017.

[42] J. Zhang, Y. Gao, Y. Jiao, L. Pu, S. Li, J. Tang, Y. Zhang, J. nanosci. nanotech., 2020, 20, 3164-3173, doi: 10.1166/jnn.2020.17391.

[43] M. Golshekan, F. Shirini,, J. Appl. Poly. Sci., 2019, 136, 48265, doi: 10.1002/app.48265.

Publisher's Note Engineered Science Publisher remains neutral with regard to jurisdictional claims in published maps and institutional affiliations. 